\title{
Climate-sensitive urban growth: outdoor thermal comfort as an indicator for the design of urban spaces
}

\author{
E. Tapias \& G. Schmitt \\ Chair of Information Architecture, ETH Zurich, Switzerland
}

\begin{abstract}
Urban climate conditions affect how cities will develop in the future, not only because of the impact on the environment or on the energy consumption of buildings, but also on outdoor human comfort. The configuration of buildings is one of the main factors that influence the different microclimates in the city. Understanding and especially being able to predict and manipulate these urban microclimates may help improve different aspects of urban life including the outdoor thermal comfort. Because of this, it is possible to use indices of outdoor thermal conform to understand when and how the configurations of building affect the microclimate conditions. This paper describes a method that; (i) enables the integration of microclimate data into the creation of new urban forms using outdoor thermal comfort as an indicator, and (ii) translates this knowledge into a parameterized design-feedback tool. In this way, it will be possible to support the design process by automated tools that explore design spaces of urban forms according to measurements and empirical findings on the relationship between microclimate data and the building geometries.

Keywords: outdoor thermal comfort, microclimate data, building geometries, generative design, physiological equivalent temperature.
\end{abstract}

\section{Introduction}

The world is facing unprecedented speeds in climate-change leading to an increase in global average temperatures, commonly referred to as global warming. Cities are seen not only as potential sites of climate vulnerability but also as the main contributor [1]. Urban areas absorb and retain significantly more heat than rural 
areas [2]. This warmth of cities in contrast to their surrounding is known as 'Urban Heat Island' or UHI. One of the most important factors affecting the intensity of the UHI is the configuration of buildings; moreover, the situation differs according to the local climate. As suggested by the 'United Nations Environment Programme', cities need to adapt to future urban climates. Understanding and especially being able to predict and manipulate urban microclimates may help improve aspects on pedestrian activity in urban spaces, and on the performance of buildings, especially in respect to energy conservation [3-5].

Awareness on how to design in accordance with the environment and the climate is increasing. Different climatic aspects such as climate conditions, seasonal variations and climate change bring additional demands on the planning and design of urban developments. Urban 'climate-sensitive' design is defined as a process that considers the fundamental elements of microclimates (e.g., sun, wind, temperature) for design purposes. This concept is applied not only to benefit from the existing urban microclimate but also to mitigate it's already stressed condition and decrease the negative effects through design and planning options. However, this concept requires a more scientific approach to evaluate its true meaning [6], which implies a method of inquiry that must be based on empirical and measurable evidence subject to specific principles of reasoning. Therefore, this paper describes a method that; (i) enables the integration of microclimate data into the creation of new urban forms using outdoor thermal comfort as an indicator, and (ii) translates this knowledge into a parameterized design-feedback tool.

The outdoor thermal comfort is generally studied on the urban micro-scale, which is affected by the Canopy layer heat island (CLHI), and can be described and linked to microclimatic conditions by steady-state assessment methods [7]. With this information and after field measurements, a 'predicting tool' can be implemented in order to simulate how changes in design details influence outdoor thermal comfort [8]. This methodology has been a reliable data source for studies in different climatic conditions [9-11].

Cities demand a high-level of city planning with knowledge on how to adapt, transform and reinvent built environments. In this sense, it is useful to consider architectural design as a particular type of problem-solving process. If the goal is to arrive at something that does not yet exists, it is possible to construct a generative system, which can then be operated to produce a variety of potential solutions [12]. In order to support city-planning processes, we need to develop tools that allow assessment of the best in order to provide solutions that help the construction of future cities [13]. Therefore, there is a need of an automated method that allows the systematic exploration and evaluation of different geometric alternatives to explore design spaces as variants of urban forms.

In this way, it will be possible to support the design process by automated tools to explore design spaces of urban forms according to measurements and empirical findings on the relationship between the thermal comfort, microclimate data and the building geometries. 


\section{Outdoor thermal comfort}

Outdoor spaces are important for cities as these provide daily pedestrian traffic and different outdoor activities contributing to urban livability and vitality [7]. Promoting the use of streets and outdoor spaces by pedestrians will benefit cities from physical, environmental, economical, and social aspects [14]. In this way, ensuring that people are comfortable in outdoor spaces is essential to a better quality of urban life. Over the past few decades, making outdoor spaces attractive to people, and ultimately used by them, has been increasingly recognized as a goal in urban planning and design [7]. Among many factors that determine the quality of outdoor spaces, the urban microclimate is an important one. Pedestrians are directly exposed to their immediate environment in terms of variations of air temperature, relative humidity, wind speed, and solar radiation. Therefore, people's sensation of thermal comfort is greatly affected by the local microclimate [7].

Urban planners intending to create comfortable microclimates can profitable from easy methods for the assessment of the thermal component of climate [11]. In the past decade, some studies on biometeorology and urban climatology for enhancing urban spaces have been focused on modelling and assessment methods from a thermo-physiological perspective [15]. The degree of impact of outdoor thermal environment on thermal comfort varies with the thermal requirements of people in different climatic regions. A number of bio-meteorological indices have been developed to describe human thermal comfort level by linking local microclimatic condition and human thermal sensation [7]. A major group of such indices are the so-called 'steady-state' models. These models are based on the assumption that people's exposure to an ambient climatic environment has, over time, enabled them to reach thermal equilibrium, and they provide numerical solutions to the energy balance equations governing thermoregulation. One of the most widely used indices is the Physiological Equivalent Temperature (PET) [16]. PET is a temperature dimension index measured in degrees Celsius $\left({ }^{\circ} \mathrm{C}\right)$, making its interpretation comprehensible to people without a great deal of knowledge about meteorology. PET is based on the Munich Energy-balance Model for Individuals (MEMI) [17] and is defined as the air temperature at which, in a typical indoor setting, the human energy budget is maintained by the skin temperature, core temperature, and sweat rate equal to those under the conditions to be assessed [11]. Although there are other 'steady-state' models that serve as analytical tools to assess human thermal responses to the local thermal environment (such as PMV, ITS, fuzzy-PMV, OUT-SET and COMFA), PET is particularly suitable for outdoor thermal comfort analysis [7,8] and is already included in the VDI (German Association of Engineers) guideline 3787 for human bio-meteorological evaluation of climates in urban and regional planning.

The outdoor thermal condition is impacted by various elements in the built environment such as; anthropogenic heat [18] ground surface covering [19] evaporation and evapotranspiration of plants [20] and shading mainly by trees and buildings. The outdoor thermal comfort is generally studied on the urban microscale level, which is affected by the Canopy Layer Heat Island (CLHI). As shade 
can block incident solar radiation, some studies have discussed mainly the shading effect on thermal environments. For example, street orientation and the height/ width $(\mathrm{H} / \mathrm{W})$ ratio have been measured to assess the shading levels in some studies $[21,22]$. In the context of urban planning, how outdoor thermal conditions influence thermal sensations of people and their behaviour (use of outdoor spaces) is of great interest for designing urban spaces.

\section{Analysis and simulation in city planning and design}

Simulation can be defined as the imitation of some real thing, a process (theory and prediction) [23] or a situation to verify, find or explain a theory. The design perspective defines simulation as the creation of past, present, or future scenarios, representing interaction of crucial parameters and variables [24].

There is a growing tendency on finding how our understanding of the urban microclimate may be applied in practice for urban planning and design. This is not an easy task as trying to respond to different criteria of urban microclimate might lead to contradictory requirements. However, microclimate has an effect on a very broad range of issues encompassed in the field of urban planning and design. The different effects of urban microclimate in urban planning and design can be divided into two groups; the effect of microclimate on the performance of buildings, especially in respect to energy conservation and the effect of microclimate on human activity, specially pedestrian, in the spaces between building [3].

\subsection{Measuring outdoor thermal comfort}

As mentioned above, there are different indices to measure the thermal comfort of pedestrians. The Physiological Equivalent Temperature (PET) is a notable example of a steady-state model. It is defined as the air temperature at which, in a typical indoor setting (air temperature $=$ mean radiant temperature, relative humidity $=50 \%$, wind speed $=0.1 \mathrm{~m} / \mathrm{s}$ ), the heat budget of the human body is balanced with the same core and skin temperature as those under complex outdoor conditions $[8,11]$. PET enables to compare the integral effects of complex thermal conditions outside with a person's own experience in indoors $[8,11]$.

PET can be measured by several computational tools such as ENVI-met, SOLWEIG, COMFA+, OTC model, and RayMan model. RayMan stands for 'radiation on the human body' and is an urban climate analysis tool that has been used in urban built-up area with complex shading patterns and generated accurate predictions of thermal environments [8]. The meteorological parameters influencing the human energy balance and needed to calculate PET in the RayMan model are; air temperature (Ta), relative humidity $(\mathrm{RH})$, wind speed (v), and global radiation (Gr). In addition, the RayMan model needs morphological and geometrical data of urban forms, personal data (human clothing and activity), location, and day and time of the year. 


\subsection{Modelling the urban microclimate}

Modelling a planned built environment project helps to visualize its future impact and is an important part of responsible design decision-making [3]. There are three types of modelling methods used in urban climatology; physical scale models, integrated open-air models, and mathematical models. Computer programmes simulate the physical environment and produce knowledge that may say something about real-world behaviour. However, all types of models need validation, and because of the deficiency of well-documented high-quality data from field studies, this remains a major problem for most urban climate models [3].

In terms of outdoor thermal comfort, there is the need for a predicting tool by means of urban climate models and tools. Although people's subjective perceptions and responses to the urban environment are various and not yet well understood, simulation and scenario-testing tools are always of particular importance in an assessment framework because they provide a platform for the integration of knowledge from various perspectives and comparisons of various design scenarios [7]. These "predicting tools" can support the research on how changes in design details influence outdoor thermal comfort. The statement applies with equal force to the more general context of research in this area, which is how urban design can influence the microclimate of an urban environment and people's outdoor thermal comfort and, in turn, how people's thermal comfort can influence their use of outdoor urban spaces. Design regulations and guidelines in this respect require comprehensive assessment before they are adopted. City planners and decision makers, when faced with the task of designing urban spaces that are desirable and thus used rather than abandoned, will be better informed with a predicting tool that allows various design alternatives to be compared and tested in terms of attractiveness and effectiveness. In particular, a testing tool is needed that can provide both quantitative and qualitative understanding of the relationships among microclimatic environment, subjective thermal assessment, and social behavior. Such a tool should have the ability to process detailed environmental information according to time and location variations and to generate analytical results to reveal the relationship. Environmental modeling tools such as Rayman can provide an understanding of climatic conditions, and can provide assessment of human thermal comfort [7].

In order to correlate the urban configurations with the thermal comfort indices, the Sky View Factor (SVF) can be implemented $[8,25,26]$. The SVF measures the ration of free sky spaces affected by geometry and according to fisheye photographs [27]. After climatic and environmental factors and fisheye photographs are imported to the RayMan model, the PET, mean radiant temperature (Tmrt), and SVF are output by the model. Fisheye photographs of the sky taken from the measurement location are imported into the 'RayMan' model to include the shading effect while calculating short- and long-wave radiation fluxes [8]. 
With this correlation established, it is possible to develop a generative method that systematically translates the results from the RayMan model into geometric alternative in order to explore design spaces.

\section{Generative systems in design and planning}

It is useful to consider architectural design as a particular type of problem-solving process in order to discuss design within the framework of a general theory of problem-solving [12]. In problem-solving if the goal is to arrive to something that does not yet exist, it is possible to construct a generative system, which can then be operated to produce a variety of potential solutions. In this context, Aristotle argued that, in a similar way, "designs for potential cities could be generated by analyzing cities into their essential constituent parts, listing the alternatives for each part, then taking various different combinations of alternatives" (Mitchell, 1977, p. 30). Generative systems have played important roles in the development of engineering and architectural design methodology. In architecture, these methods are based upon systematic exploration of alternative ways in which various elements from a fixed vocabulary could be assembled in different combinations to generate architectural forms. This systematic use of generative systems in architectural design can also be applied in urban design and planning as Aristotle ones proposed.

Within a generative system, it is important to define the representations of potential solutions, the identification of the acceptable solutions, the solution generation procedures, and the solution evaluation techniques [12].

When a generative system 'represents' potential solutions to a design problem, it means that each particular potential state of the system clearly represents one specific potential solution (state-action graph). Therefore, the construction of the system indirectly establishes a set of potential solutions for exploration. A system can be symbolically model (symbolic representation) by letting specific design variables represent specific properties of the system.

For the identification of acceptable solutions with a generative system, first it is important to clarify if there is a clear problem. A 'well-defined' problem can be recognized if there is a test that can identify a solution. A 'set-predicate description' can be used as a test by first defining a goal set. In this way, we can identify three types of set of solutions; the under-specified (many satisfactory solutions), the uniquely-specified (only one satisfactory solution), and overspecified (no satisfactory solutions). When the situation is over-specified, the goal set needs to find possible solutions (goal set members) by means of constraints. A part from establishing constraints, a second common method for specifying the design criteria that identifies the goal set is to state objectives to be minimized or maximized [12].

A solution generation procedure may be thought of as a strategy for choosing particular paths through the state-action graph of a problem in search of satisfactory solutions (goal set members). For this, a 'search procedure' can be apply where require some kind of search of alternative paths through the stateaction graph (search space). The simplest kinds of search procedures are 
exhaustive enumeration of alternatives or random selection of alternatives. More sophisticated search procedures make use of some kind of information about the particular problem before hand to guide the search for a solution. The better the guiding information, the more efficient the search procedure is likely to be. If a procedure leads with complete certainty to a good solution or else demonstrates that the goal set has no goal set members at all, then it is referred as algorithm. A procedure that generates a good solution more quickly or cheaply than another has greater heuristic power [12].

Unless a solution generation procedure can be proved only to produce members of the goal set, it is necessary to employ some kind of test procedure (solution evaluation techniques) to determine whether in fact a proposed solution that has been generated is a member of the goal set [12]. The mathematical model approach requires that constraints and objectives be expressed as functions of the design variables. The forms of these functions depend upon the rules that direct the behavior of the system under consideration. Mathematical models are often categorized as either static or dynamic (time), and either deterministic or stochastic (fixed or random). A further categorization distinguishes analytical and simulation models [12].

A design problem can be characterized in terms of: data structure (variables describing the relevant properties of potential solutions), set of operators (applied to variables to change the state of the data structure), set of constraints and/or objectives (define a goal set of acceptable solutions), and solution generation procedure (set of potential solutions for consideration) [12].

\section{Approach and method}

The aim of this method is establish the relationship between, microclimate data and building geometries using outdoor thermal comfort as an indicator, and to translate this knowledge into a parameterized design-feedback tool.

After selecting distinctive urban canyon areas based on the difference on building morphology and geometry, the first objective is to calculate and compare the outdoor thermal comfort during the different seasons, and predict long-term thermal comfort by fitting local climate. The second is to correlate the outdoor thermal comfort with the building geometries. Finally, the third objective is to develop a generative method based on evolutionary design that allows the systematic exploration and evaluation of different geometric alternatives to explore design spaces (solutions) of urban forms. To achieve the aforementioned research objectives, the method is divided into three sections (Figure 1). The first one is based on employing the 'Physiological Equivalent Temperature' (PET) steady-state assessment method as a thermal index. Using the latter results, the second section is focused on the implementation of the Rayman model as the 'predicting tool' for long-term thermal comfort. In order to validate the model, previous data acquisition of meteorological parameters will be conducted in order to compare measurement and model results. After comparing the results with 'Sky View Factor' analysis, the last work package aims to develop a computational 
generative method based on evolutionary design allowing a systematic exploration of different geometric alternatives.

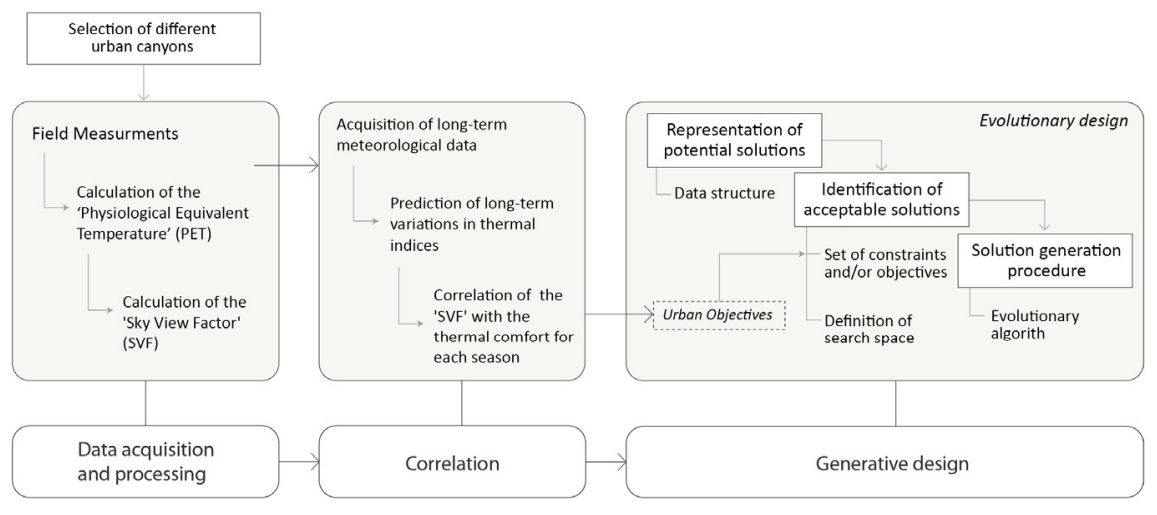

Figure 1: Method scheme.

\subsection{Measuring the physiological equivalent temperature}

Before measuring the PET, three to five different urban canyon areas will be chosen based on the difference on building morphology and geometry. After these areas are establish, portable mini weather stations are placed on a significant height of $1.1 \mathrm{~m}$ above ground. The meteorological data acquire includes; air temperature $(\mathrm{Ta})$, relative humidity $(\mathrm{RH})$, global radiation $(\mathrm{Gr})$, vapour pressure $(\mathrm{Vp})$ and wind velocity (w). Adding to these measurements, field observations on people's cloths and activity need to be recorded. These measurements are recorded at 1-minute intervals automatically from 8:00 to 18:00 and are conducted during the two seasons of the savanna tropics (humid and dry). The next step is to compare field measurement results for each location and each day with simulation results to validate the accuracy of the RayMan model. The optimal setting for the model that fits the local climate and environment is then obtained [8]. In this way, the PET can be calculated using the RayMan model.

In addition, Fisheye and street photographs of each of the selected urban areas are taken in order to calculate the SVF from the fisheye images and compare them with the PET values.

\subsection{Long-term thermal simulation}

According to the optimal settings validated in the previous work package, longterm meteorological data from weather stations are imported into the RayMan model in order to predict long-term variations in thermal environment and thermal indices. For this, the Ta, RH, v, Gr and Cd of hourly data are imported in the RayMan model. If the meteorological data are not acquire in the surveyed areas and cannot directly represent the thermal conditions at measurements locations, it can be assumed that the thermal conditions at these locations are related to 
meteorological data and data must be corrected before being imported to the model. In order to correct the data, the most similar selected area to the area at the weather station is chosen as the reference. Then, long-term Ta, v and Gr for each of the other locations are modified by climate data of the weather station based on regression analysis [8]. The previous results are used to assess the shading effect on annual thermal comfort. For these, the SVF are correlated with the thermal comfort for each season.

\subsection{Development of the generative method}

The last section of the method is intended used the previous correlation to develop a generative method that systematically explores design solutions of building geometries to enhance the outdoor thermal comfort. For the creation of a generative system it is important to define the following steps; the representations of potential solutions, the identification of the acceptable solutions, and the solution generation procedures.

\subsubsection{Representation of potential solutions}

When a generative system 'represents' potential solutions to a design problem, it means that each particular potential state of the system clearly represents one specific potential solution. Therefore, the construction of the system indirectly establishes a set of potential solutions for exploration. If a structure is a relationship between the individual elements of a set, a data structure is a collection of variables associated in some specific way. The concepts of a design variable, and of the association of the design variables into data structures, are central to an understanding of the process of design by use of symbolic generative systems.

It is important to focus on the criteria that can be measured (quantitative aspects) in order to assign them as design variables. The definition of an urban form can have a great number of degrees of freedom (i.e. parameters that that completely describe a geometry). There is a clear compromise between the level of detail (number of variable parameters) that can be included in the optimization and the complexity of the algorithm. For instance, a building form can be modelled simply as a geometric box. In this case it would be possible to completely describe its geometry by only three parameters (height, width and length). This rather simple model would be easier to implement than a more complex one, nevertheless, lacks the possibility of creating more realistic urban forms (a more complex model would allow to have other geometric alternatives rather than only a box). There is a clear tradeoff between how realistic the urban forms can be and the complexity of the optimization algorithm. Different alternatives of parameterization will be explored in this part of the work package.

\subsubsection{Identification of the acceptable solutions}

The different variables are divided into two parts; the variables from the urban objectives according to existing city planning strategies, and the variables from the results of the correlation established in the previous section. 
When the situation in a design problem is over-specified, the goal set needs to find possible solutions (goal set members) by means of constraints (defining boundaries) and/or objectives (minimized or maximized). This will allow specifying the design criteria that identify the goal set and thereby defines the search space. The search space is a space filled with all possible solutions to the problem, and a point in that space defines a solution. The different constrains and/or objectives for this specific problem are established from the resulting variables. The outcomes will also help defining the fitness within the solution generation procedure. The 'fitness' of a solution is a score based on how well the solution fulfills the problem objective, calculated by a 'fitness function', which would take a solution as input and return a fitness value based on how well the solution satisfies these objectives.

\subsubsection{Solution generation procedures}

A solution generation procedure may be thought of as a strategy for choosing particular paths in search of satisfactory solutions (goal set members). The simplest kinds of search procedures are exhaustive enumeration of alternatives or random selection of alternatives. Therefore, evolutionary design techniques will be implemented for this specific design problem. Evolutionary design builds on analysis software tools by actually taking over part of the design process and allowing automatically improving the performance of the designs [28]. More specific choice is the Integral Evolutionary Design, which evolves new designs from scratch, and optimizes them, such that they fulfill specific functional criteria.

It is necessary to understand that evolutionary computation is all about search. Evolutionary search algorithms are inspired and based upon evolution in nature. These algorithms typically use an analogy with natural evolution to perform search by 'evolving' solutions to problems. Also, these algorithms consider a large collection or 'population' of solutions at once in the search space instead of working with one solution at a time. By considering the search space, it is possible to get an idea of how evolution finds good solutions but it requires guidance to direct evolution towards better areas of the search space. They receive this guidance by 'evaluating' every solution in the 'population', to determine its 'fitness'.

\section{Conclusion}

The main outcome of this method is an automated tool to explore design spaces of urban forms according to measurements and empirical findings on the relationship between the outdoor thermal comfort, the microclimate conditions and the building geometries. This generative method will systematically translate urban climate data into design variants. In this way, this process will contribute to urban planning practices as a decision-support tool to provide guidelines for the construction of 'climate-sensitive' urban forms. The reduction of energy consumption of buildings can be a side contribution.

Although the calculation of the PET has been implemented in various different locations [7-11, 19, 22, 25, 26], it has been barely used in extreme climates such 
as the tropics. In these climates, the outdoor thermal comfort is one of the main parameters evaluated for the quality of urban life. This human comfort in the open spaces affects the pedestrian activities altering the liveability of the urban area.

\section{References}

[1] Bulkeley, H. \& Castán Broto, V. Government by experiment? Global cities and the governing of climate change. Transactions of the Institute of British Geographers 38: 361-375, 2013.

[2] Oke, T.R. The distinction between canopy and boundary layer urban heat islands. Atmosphere 14: 268-277, 1976.

[3] Erell, E., Pearlmutter, D. \& Williamson, T. Urban Microclimate: Designing the spaces between buildings. Earthscan, UK, 2011.

[4] Kolokotroni, M., Giannitsaris, I. \& Watkins, R. The effect of the London urban heat island on building summer cooling demand and night ventilation strategies. Elsevier: Solar Energy 80(4): 383-392, 2006.

[5] Santamouris, M. Environmental Design of Urban Buildings: An Integrated Approach. Earthscan, UK, 2006.

[6] Berger, M. The Unsustainable City. Sustainability 6(1): 365-374, 2014.

[7] Chen, L. \& Ng, E. Outdoor thermal comfort and outdoor activities: A review of research in the past decade. Elsevier: Cities 29: 118-125, 2011.

[8] Lin, T.P. Thermal perception, adaptation and attendance in a public square in hot and humid regions. Building and Environment 44: 2017-26, 2009.

[9] Kántor, N. \& Unger, J. Benefits and opportunities of adopting GIS in thermal comfort studies in resting places: An urban park as an example. Elsevier: Landscape and Urban Planning 98: 36-46, 2010.

[10] Nikolopoulou, M., Baker, N. \& Steemers, K. Thermal comfort in outdoor urban spaces: understanding the human parameter. Elsevier: Solar Energy 70(3): 227-235, 2001.

[11] Höppe, P. The physiological equivalent temperature - a universal index for the biometeorological assessment of the thermal environment. Int $\mathrm{J}$ Biometeorol 43: 71-75, 1999.

[12] Mitchell, W. J. Computer Aided Architecture Design. Van Nostrand Reinhold Company, Inc., USA, 1977.

[13] Grimmond, CSB, Roth, M., Oke, TR., Au, YC., Best, M., Betts, R., Carmichael, G., Cleugh, H., Dabberdt, W., Emmanuel, R., Freitas, E., Fortuniak, K., Hanna, S., Klein, P., Kalkste in, LS., Liu, CH., Nickson, A., Pearlmutter, D., Sailor, D. \& Voog, J. Climate \& More Sustainable Cities: Climate Information for Improved Planning \& Management of Cities (Producers/Capabilities Perspective) World Climate Conference - 3 better climate information for a better future, WCC3; Procedia Environmental Sciences 1: 247-274, 2010.

[14] Hakim, A., Petrovitch, H., Burchfiel, C. M., Ross, G. W., Rodriguez, B. L., White, L. R., Yano, K. \& Curb, J. D. Effects of walking on mortality among nonsmoking retired men. N. E. Journal of Medicine 338: 94-99, 1998. 
[15] Höppe, P. Different aspects of assessing indoor and outdoor thermal comfort. Energy and Buildings 34(6): 661-665, 2002.

[16] Mayer, H. \& Höppe, P. Thermal comfort of man in different urban environments. Theor. Appl. Climatol. 38: 43-49, 1987.

[17] Höppe, P. Die Energiebilanz des Menschen. Wiss. Mitt. Meteorol. Inst. Uni München 49, 1984.

[18] Ichinose, T., Shimodozono, K. \& Hanaki, K. Impact of anthropogenic heat on urban climate in Tokyo. Atmospheric Environment 33: 3897-909, 1999.

[19] Lin, TP., Ho, YF. \& Huang, YS. Seasonal effect of pavement on outdoor thermal environments in subtropical Taiwan. Elsevier: Building and Environment 42: 4124-31, 2007.

[20] Robitu, M., Musy, M., Inard, C. \& Groleau, D. Modeling the influence of vegetation and water pond on urban microclimate. Solar Energy 80: 43547, 2006.

[21] Emmanuel, R., Rosenlund, H. \& Johansson, E. Urban shading - a design option for the tropics? A study in Colombo, Sri Lanka. International Journal of Climatology 27: 1995-2004, 2007.

[22] Johansson, E. Influence of urban geometry on outdoor thermal comfort in a hot dry climate: a study in Fez, Morocco. Building and Environment 41: 1326-38, 2006.

[23] Hartmann, S. The World as a Process. Simulations in the Natural and Social Sciences, in Hegselmann et al. 1996, 77-100, 1996.

[24] Lenhard, J., Küppers, G. \& Shinn, T. Simulation: pragmatic construction of reality. Springer, 2006.

[25] Lin, T.P., Matzarakis, A. \& Hwang, R.L. Shading effect on long-term outdoor thermal comfort. Building and Environment 45: 213-221, 2010.

[26] Hwang, R.L., Lin, T.P. \& Matzarakis, A. Seasonal effects of urban street shading on long-term outdoor thermal comfort. Elsevier: Building and Environment 46: 863-870, 2010.

[27] Svensson, M.K. Sky view factor analysis - implications for urban air temperature differences. Meteorol. 201-211, 2004.

[28] Bentley, P.J. Evolutionary design by computers. Morgan Kaufmann, USA, 1999. 